\title{
Mort d'un héros. Tensions politiques autour de l'enterrement de Bienvenu Roland Faignond à Brazzaville
}

\section{Marc-Eric Gruénais}

\section{(2) OpenEdition Journals}

Édition électronique

URL : http://journals.openedition.org/apad/213

DOI : 10.4000/apad.213

ISSN : 1950-6929

Éditeur

LIT Verlag

\section{Édition imprimée}

Date de publication : 1 juin 2003

\section{Référence électronique}

Marc-Eric Gruénais, « Mort d'un héros. Tensions politiques autour de l'enterrement de Bienvenu Roland Faignond à Brazzaville », Bulletin de l'APAD [En ligne], 25 | 2003, mis en ligne le 15 juin 2007, consulté le 08 septembre 2020. URL : http://journals.openedition.org/apad/213 ; DOI : https://doi.org/ 10.4000/apad.213

Ce document a été généré automatiquement le 8 septembre 2020

Bulletin de I'APAD 


\title{
Mort d'un héros. Tensions politiques autour de l'enterrement de Bienvenu Roland Faignond à Brazzaville
}

\author{
Marc-Eric Gruénais
}

1 IRD - janvier 2004

2 Le soir du 15 janvier 2004, Brazzaville est en émoi : le corps de Bienvenu Rolant Faignond, maire de l'arrondissement 3 de Brazzaville (Poto-Poto), blessé par des "balles perdues", est mort à Paris où il avait été évacué. Quelques jours auparavant, la nouvelle était tombée: Faignond, blessé le 18 décembre 2003 dans son quartier de Poto-Poto, évacué en France pour être soigné, a succombé à ses blessures. Comme de coutume au Congo, et étant donné la notoriété du personnage, une immense veillée funèbre avait été organisée dans son quartier dès que l'information avait été connue. Toute la bonne société brazzavilloise s'y pressait, pour se montrer, y rencontrer des amis, rendre hommage au grand homme, présenter ses condoléances à la famille. Mais la "mauvaise herbe" brazzavilloise, composée des jeunes du quartier Poto-Poto, était également présente. La tension à la veillée était palpable : des forces de sécurité sont présentes ; elles dispersent parfois évacuer sans ménagement des regroupements qui s'organisent lors de la veillée, peut-être de crainte de débordements. A qui veut l'entendre, chacun conseille à son voisin de ne pas rester trop tard dans Brazzaville hors de chez soi tant que l'enterrement n'aura pas eu lieu.

3 Depuis les guerres civiles, qui ont secoué le pays de 1993 à 2000, et jusqu'en cette année 2004 , les Brazzavillois ont peur, et la rumeur va bon train. Tout événement politique est potentiellement source de dérapage et de violence.

4 Le 15 janvier au soir, la dépouille de Bienvenu Faignond arrive par le vol Air France à l'aéroport de Maya Maya. Le peuple de Brazzaville se masse sur les voies que le cercueil doit emprunter pour être conduit à la morgue. Les media congolais retransmettent en direct la procession. Le lendemain, tout Brazzaville, en deuil, retient son souffle. 
Bienvenu Roland Faignond, qui venait d'avoir 53 ans, sera enterré ce jour au cimetière $\mathrm{du}$ centre ville, cimetière réservé aux personnalités politiques. Le matin de l'enterrement, toute la ville, et en particulier, les chauffeurs de taxis, préoccupés des risques de perturbation de la circulation et/ou de débordements, étaient dans l'ignorance du trajet qu'emprunterait le cercueil avant son inhumation : irait-il aussi à l'Hôtel de ville central ? à Poto-Poto ? Une messe de requiem est organisée au Palais du Parlement et retransmise en direct à la télévision. Toute la classe politique est présente à cette cérémonie, à commencer bien évidemment par le Président de la République lui-même, Denis Sassou Nguesso, qui décorera B.R. Faignond à titre posthume. Les homélies des caciques du régime se succèdent pour vanter les mérites de ce grand Congolais. Pour la circonstance, tous les marchés de la capitale congolaise ont été fermés, en signe de deuil national. Après cette cérémonie, le cercueil fut immédiatement emmené au cimetière du centre ville, comme si le Pouvoir avait hâte de procéder à l'inhumation ; les craintes des Brazzavillois et des chauffeurs de taxi étaient donc infondées.

5 Mais les "petits du quartier", les "enfants de Poto-Poto", ont été fort mécontents quant à la manière dont la cérémonie funèbre a été organisée par le Pouvoir. Le journal L'Observateur faisait remarquer que la messe de requiem avait été prononcée au Palais du Parlement, lieu emblématique du régime en place, et non en la basilique Sainte Anne, ce monument de l'arrondissement du maire décédé. Manifestement, les "enfants de Poto-Poto" espéraient que la dépouille de leur champion fut exposée "chez lui", dans "son" quartier, ou au minimum dans cette basilique Sainte Anne située à l'entrée du quartier de Poto-Poto. Les "enfants de Poto-Poto", entendait-on dire, ont "boudé" l'enterrement (un nombre très réduits de leurs représentants se seraient rendus au Palais du Parlement). D'aucuns ont prétendu que des sommes d'argent leur auraient été distribuées pour les "calmer". Mais, même très contrariés, ils ne pouvaient cependant pas laisser totalement la dépouille de leur leader aux mains du Pouvoir. Ce sont des "enfants de Poto-Poto" qui auraient obtenu de porter le cercueil de leur champion, à pied, du Palais du Parlement jusqu'au cimetière du centre ville. Mais chacun de se demander, après l'enterrement, ce qu'allaient devenir ces "enfants", désormais orphelins, après le décès de leur chef?

Qui était Bienvenu Faignond? La meilleure définition de ce personnage, dans laquelle tous les Brazzavillois se retrouvent, est de le présenter comme un véritable "enfant de Poto-Poto". Poto-Poto, rappelons-le, est l'un des quartiers historiques du centre ville de la capitale congolaise : quartier d'accueil des migrants venant de tout le Congo, mais aussi de l'AEF et de l'Afrique de l'Ouest, lieu de résidence de travailleurs du chemin de fer Congo Océan durant la période coloniale, lieu aussi par excellence de tous les "plaisirs" de Brazzaville, avec ses bars, sa musique, ses lieux de prostitution, etc. Poto-Poto est traditionnellement opposé à cet autre quartier historique de la capitale congolaise, Bacongo, plus "sage", plus homogène dans son peuplement, dont on dit qu'il rassemble essentiellement des ressortissants des régions du sud du pays (Lari et Bakongo). Le père de Bienvenu Faignond, un métis, décédé il y a deux ans, est notamment connus comme étant le fondateur de l'Espace Faignond, bar, hôtel, boîte de nuit, qui au début des années d'Indépendance, était un haut lieu de la vie nocturne brazzavilloise, où se retrouvait notamment quelques grandes dames, certaines revendiquant leur statut de "femmes libres". Si l'Espace Faignond a aujourd'hui un peu perdu sa superbe, il s'était maintenu notamment grâce à Bienvenu Faignond, le fils, qui a repris l'affaire familiale. Faignond était surtout devenu une figure charismatique de 
Poto Poto. Il arborait des tenues vestimentaires que l'on ne s'attend guère à voir porter par un maire. Grand amateur de football, il organisait notamment les bandes de jeunes de son quartier de Poto-Poto. Il avait accru considérablement sa notoriété en créant un groupe musical, 'Bana Poto Poto' ("Les enfants de Poto Poto") ${ }^{1}$. Chanteur et compositeur, Faignond a remporté un immense succès avec la chanson qu'il interprétait lui-même intitulée 'matiti mabe' ("mauvaise herbe"), qui "fait allusion au chanvre indien consommé par les jeunes à Brazzaville...". Cette chanson passait d'ailleurs "en boucle" sur les antennes de la radio et de la télévision les 15 et 16 janvier, pour saluer la mémoire de ce maire, apparemment très atypique au sein d'une élite politique habituellement plutôt guindée.

7 Mais Bienvenu Faignond n'était pas un "enfant de Poto Poto" ordinaire. Docteur en droit, il avait assuré de hautes responsabilités dans le secteur des hydrocarbures dont on sait qu'il s'agit là de la principale ressource du pays. Le Journal L'Observateur rappelait qu'il avait été conseiller juridique auprès du PDG d'Hydro Congo (la société nationale gérant le pétrole congolais et aujourd'hui disparue), puis du ministre des Hydrocarbures. Mais surtout, la rumeur présente Bienvenu Faignond comme un fervent soutien de Denis Sassou Nguesso durant la guerre civile de 1997 : on rapporte qu'il avait été à la tête d'un groupe armé, appelé le Front 400, qui aurait combattu aux côtés des troupes de Sassou Nguesso qui repartait alors à la conquête du pouvoir contre son grand rival de l'époque, Pascal Lissouba, élu Président de la République en 1992. La rumeur (toujours elle !) rapporte que Bienvenu Faignond aurait été nommé maire, puis reconduit en 2003 dans ses fonctions à la tête de "son" quartier, Poto-Poto, en récompense de ses faits d'arme.

Les versions des circonstances de sa mort divergent. Le journal La Rue Meurt rapportait que Faignond avait été touché par des "tirs meurtriers de braqueurs en pleine opération à Poto-Poto"; le journal Tam-Tam d'Afrique évoquait pour sa part des "assaillants qui venaient selon diverses sources des quartiers Nord de Brazzaville [fief des partisans de Sassou Nguesso], conscients que l'attention de la force publique était focalisée sur les quartiers sud, [qui] avaient tenté de mettre à sac les magasins de Poto-Poto et Moungali". L'Observateur précisait que Faignond avait été atteint par balle au cou et à l'abdomen le 18 décembre 2003 lors d'une intervention de police contre les pilleurs d'un magasin au marché de Moungali. La version du journal L'Humanitaire est légèrement différente : Faignond, "victime en décembre d'une bavure policière lors des troubles qui avaient secoué le sud de la capitale congolaise, a succombé à ses blessures samedi à Paris [10 janvier 2004], où il recevait des soins intensifs... C'est en faisant la ronde de son quartier [Poto-Poto] pour prévenir des actes de pillage, le 17 décembre dernier, qu'il avait été atteint de plusieurs balles de kalachnikov de la brigade antigang à la poursuite d'un groupe de voleurs armés. Ces voleurs avaient pris la fuite à bord d'un véhicule Toyota semblable à celle du maire". La version d'une erreur de la brigade anti-gang tirant sur la voiture du maire en pensant que se trouvaient à bord, non pas Faignond lui-même, mais les voleurs de sa voiture peut évidemment donner lieu à toutes les interprétations. La rue, et surtout les "enfants de Poto-Poto" qui combattaient pour Sassou Nguesso en 1997 sous l'égide de Faignond, se demandent alors évidemment si le décès de leur leader a été vraiment dû à une bavure.

Pour comprendre les enjeux liés à la mort de Faignond, il faut opérer un retour en arrière. Depuis 1993, dès les premiers mois de la période dite de "démocratisation", Brazzaville a été le théâtre de violents affrontements entre différents "champions" 
désireux de gouverner le pays. Ces rivalités ont d'abord culminé avec la guerre civile de 1997 qui a placé sur le devant de la scène les différentes milices des principaux leaders, et a permis le retour au pouvoir de Denis Sassou Nguesso. Un second pic de violences a lieu en 1999, lorsque les troupes de Sassou Nguesso tentent de venir à bout d'une des milices encore fortement armées et qui dénient toute légitimité au nouveau président. Cette nouvelle guerre civile de 1999 ne débouche guère sur une victoire de Sassou Nguesso mais plutôt sur un statu quo où les positions de Sassou Nguesso et de ses opposants armés sont entérinées. Depuis 2000 on assiste à une tendance à la pacification, avec cependant des échauffourées régulières, qui attestent de l'importance de l'armement dont disposent toujours les différents groupes armés présents dans le pays.

10 C'est dans ce contexte de paix fragile que les 15,16 et 18 décembre 2003, les quartiers sud de Brazzaville (Bacongo et Makélékélé) sont de nouveau le théâtre de tirs à l'arme lourde. La première version officielle présentée précise qu'il s'agit là d'une dispute de miliciens sudistes, donc hostiles à Sassou Nguesso, autour de distribution de nourriture. Les quartiers sud de la capitale (Bacongo et Makélékélé) avaient été durement touchés durant la dernière guerre de 1999 ; des camps de réfugiés et des distributions d'aide alimentaire y avaient été organisés. Il n'y aurait eu rien d'étonnant qu'en décembre 2003, à la veille des fêtes de fin d'année, des groupes rivaux d'une même milice s'affrontent pour s'accaparer des vivres de l'aide alimentaire pour passer des fêtes de Noël et de Nouvel an dans de bonnes conditions avec leur famille! Cette première version aurait pu être crédible si le leader de l'opposition armée du sud n'avait pas opposé un vigoureux démenti, faisant porter la responsabilité de ces affrontements au Gouvernement: il accusait le régime Sassou Nguesso d'avoir voulu attaquer ses combattants, violant ainsi de fait l'accord de cessez-le-feu conclu le 17 mars 2003 entre ses troupes et le Gouvernement. La thèse de la dispute de bandes rivales au sein du même camp n'étant plus crédible, dans un second communiqué, le Gouvernement congolais admettra que les troubles étaient dus à "des éléments incontrôlés de la force publique et à des ex-combattants", reconnaissant ainsi en partie l'implication de forces gouvernementales dans les combats. Ainsi, du règlement de comptes local entre des jeunes pilleurs, on est donc passé à la thèse d'un nouveau conflit entre des forces "incontrôlées" liées au régime en place et la milice de l'opposition.

11 En fait il semble que les événements de la mi-décembre 2003 auraient pu faire vaciller le pouvoir. Durant la guerre de 1997, dans les parties est (dont Poto Poto) et nord de Brazzaville, favorables à Sassou Nguesso, des groupes armées "Cobra" 2 se sont organisés dans différents quartiers de cette zone sous la direction de chefs de guerre. Faignond aurait donc été l'un d'entre eux. A propos des événements de la mi-décembre 2003, reconnus donc comme étant aussi dus à des groupes incontrôlés des forces de sécurité du régime, "on" rapporte que des ex-Cobras, donc initialement favorables à Sassou Nguesso, seraient partis des quartiers les plus septentrionaux de la ville, auraient traversé toute la capitale non sans désarmer quelques gardes du corps des caciques actuelles du régime et s'emparer de leurs véhicules, pour se rendre à Bacongo et tenter de faire alliance avec les Ninjas, la milice sudiste opposé à Sassou Nguesso, pour déstabiliser le régime en place. Les Ninjas, qui contrôlent le quartier de Bacongo désormais pacifié, alors peu convaincus par les "bonnes intentions" de leurs ennemis d'hier ${ }^{3}$, et craignant surtout des représailles meurtrières en cas d'échec de ce que certains ont pu qualifier de tentative de coup d'Etat avorté, ont repoussé vigoureusement les Cobras. Ceux-ci seraient alors allés faire "le coup de feu" à 
Poto-Poto, ce qui aurait été fatal à Faignond. Cependant, la question de savoir si la mort de Faignond était une bavure ou une élimination a été posée.

12 A la suite de ces événements de décembre 2003, le régime en place organisait l'Opération Espoir pour mettre fin aux débordements des "bandes armées". L'allocution du Président Sassou Nguesso le 31 décembre 2003 devant des officiers laissait peu de place à l'équivoque. Le journal L'Autre-Vision, reprenant un communiqué officiel ${ }^{4}$, rapportait les propos suivants du chef de l'Etat: "Tous les éléments véreux égarés qui n'ont pas bien choisi leur carrière et qui se sont retrouvés comme par hasard dans la force publique doivent être mis sans ménagement hors de cette dernière et avec fermeté... l'opération Espoir devra mettre hors d'état de nuire tous ceux des éléments de la force publique égarés qui pourraient s'attaquer aux libertés des autres citoyens et à leurs biens, à leur intégrité physique". Qui sont ces "éléments véreux" qu'il faut mettre "hors d'état de nuire" ? Il y a certes la désormais "traditionnelle rébellion" des originaires des régions du Sud, et composée des Ninjas, mais aussi et surtout ces "combattants" originaires du nord, qui ont porté Sassou Nguesso au pouvoir en 1997, et qui ont été recrutés dans l'armée et la police.

13 C'est donc par le cercle de ses propres clients, soit les ressortissants du nord du pays, que le régime en place serait menacé. Le journal, le Défi Africain n'hésitait pas à rapporter des rumeurs relatives à une "prétendue rébellion du nord", qui aurait été dirigée par un certain colonel Eyoma dont la "neutralisation [dans le cadre de l'Opération Espoir]... aurait été consécutive aux événements qui ont secoué les quartiers sud de Brazzaville [en décembre 2003]" et qui "serait le cerveau de ces troubles à la veille des fêtes du Nouvel an". Dans un autre article de ce même journal on apprenait qu'une vingtaine de bandits de Talangaï (quartier de Brazzaville traditionnellement favorable à Sassou Nguesso) avaient été arrêtés dans le cadre de l'opération Espoir, en réaction au banditisme "qui a renforcé l'insécurité dans différents quartiers de Brazzaville, dont Talangaï, considéré jusqu'à une certaine date comme quartier des intouchables du pouvoir". Est-ce à dire que ce sont des combattants proches de Sassou Nguesso qu'il faut mettre "hors d'état de nuire" ? Un article de $L$ 'Observateur intitulé "Le pouvoir de Brazzaville continue de manger ses enfants", évoquant l'Opération Espoir, posait les questions suivantes : "Il se passe bien quelque chose dans le camp de Sassou Nguesso". La guerre étant finie et gagnée pour les Forces Démocratiques et Patriotiques [la coalition politico-militaire qui a permis la restauration de Sassou Nguesso à la tête du pays], donc mission terminée pour les guerriers qui les ont aidés à reconquérir le pouvoir en 1997. Elles procèdent aujourd'hui à l'élimination classique et chirurgicale, ou à la traque des ombres gênantes et voraces que sont devenus les ex-guerriers. Au commencement, il y a eu 'Chien méchant', puis vinrent les tours de Willy Mantsanga, Eyoma [noms de guerre des chefs de milices pro-Sassou Nguesso], "et peut-être même Roland Bienvenu Faignond" (souligné par moi). Le soupçon de l'élimination par le pouvoir en place du chef de guerre Faignond est donc lancé. Il convient ici de rappeler que chaque chef de guerre favorable à Sassou Nguesso possédait, et possède vraisemblablement encore, une clientèle armée, et contrôlant différents secteurs de la ville; aujourd'hui, le pays se reconstruit lentement et est contraint à une "normalisation" à laquelle les jeunes miliciens qui tirent leur statut de leurs armes risquent de ne trouver aucun avantage. En fait, le décès de Faignond et les interrogations qu'il suscite sont révélateurs de la tension larvée qui règne toujours à Brazzaville, dans un contexte de paix certes revenue depuis 2002, mais qui reste encore très fragile, d'autant plus que le régime en place doit prendre des mesures importantes 
et impopulaires pour relancer le pays, et que la responsabilité de ceux qui ont porté Sassou Nguesso au pouvoir dans diverses exactions apparaît au grand jour.

Ainsi, un rapport d'Amnesty International daté de 2002 reconnaissait que toutes les parties en conflit (donc y compris les partisans de Sassou Nguesso) étaient responsables "de graves atteintes aux droits humains fondamentaux, homicides illégaux commis sans discrimination, 'disparitions', viols et autres mauvais traitements". Aujourd'hui, "l'Affaire des disparus du Beach" continue de défrayer la chronique: des dizaines de Congolais quittant Kinshasa - où ils avaient trouvé refuge pour échapper aux combats de 1997 et 1999 - pour rejoindre Brazzaville auraient disparu alors qu'ils accostaient sur la rive brazzavilloise du fleuve, et une plainte a été déposée par les familles des victimes. De plus, à l'Opération Espoir évoquée ci-dessus, s'ajoute désormais le lancement, au début de l'année 2004, de l'opération "Démobilisation, Désarmement et Réinsertion" soutenue par le PNUD et s'adressant aux ex-combattants de tout bords. L'enjeu pour les ex-combattants est évidemment de savoir qui sera "réinséré" et qui ne le sera pas. Tout un chacun semble être prêt à négocier pour obtenir un poste et un salaire dans l'armée ou la police. Par exemple, le pasteur Ntoumi, l'actuel chef de la rébellion du Sud, qui se trouverait encore dans le maquis, qui pose comme condition de son retour à Brazzaville non seulement l'intégration de "ses" combattants dans l'armée et la police, mais aussi sa nomination "à un poste budgétaire" en tant que "général instructeur spirituel des forces armées congolaises"! Des ex-Cobras, favorables à Sassou Nguesso, ou des ex-Nin jas, milice de la rébellion du sud, qui sera "réinséré" en priorité ?

15 Mais au-delà de la mise en cause du régime et des incertitudes quant au devenir des ex-combattants, il faut aussi prendre en compte la mauvaise situation économique du Congo. Nombre d'entreprises d'Etat ont disparu, ou sont en voie de disparition pour cause de privatisation; mais leurs salariés n'ont pas pour autant disparu, et réclament leurs arriérés de salaires (non versés pendant les guerres) et/ou leurs indemnités de licenciements. Les investisseurs tardent à revenir au Congo : les coupures d'électricité et les difficultés de transport dans le pays, en dehors de problèmes d'insécurité, rendent les matières premières congolaises très onéreuses dans le contexte international d'un marché du bois et des hydrocarbures, principales ressources du pays, très concurrentiel. Le Congo Brazzaville, depuis le premier règne de Sassou Nguesso (1979-1989), a toujours été très endetté ; et les guerres qui se sont succédé de 1993 à 2000 ont évidemment encore aggravé la situation. La dette extérieure et intérieure du pays est considérable, et il ne saurait y avoir "normalisation", c'est-à-dire reprise de l'aide et des investissements, sans manifestation de bonne volonté du pays par un début de remboursement de ses dettes. D'autant que le Congo dégage des excédents pétroliers ${ }^{5}$ sur lesquels le FMI comptent beaucoup pour alléger le service de la dette, une des conditionnalités pour parvenir à un accord qui tarde à être signé. Or, on peut aisément comprendre les hésitations du régime congolais à utiliser les recettes pétrolières pour rembourser ses dettes extérieures. Accepter les conditionnalités des institutions financières internationales pour une reprise de l'aide au développement, c'est non seulement rembourser la dette, mais aussi, entre autres, poursuivre les privatisations des services publics (transports fluviaux, portuaires et ferroviaires ; eau, électricité, postes, téléphone, notamment) et "nettoyer" les fichiers d'une fonction publique pléthorique dans certains secteurs ${ }^{6}$. C'est donc augmenter le nombre de mécontents et de prétendants à des indemnités de licenciement. Le régime doit aussi faire face aux aspirations d'une jeunesse qui a accumulé des années de retard dans sa 
scolarité, qui continue d'aspirer à une formation dans des établissements scolaires et universitaires; or ces établissements ont été pillés et manquent cruellement d'enseignants, du fait de décès, de départs à la retraite, ou de départ à l'étranger. Aussi, ne serait-ce que dans le secteur de l'éducation (on pourrait aussi bien évidemment évoquer le secteur de la santé), les besoins en équipements et en ressources humaines sont très importants, et cela dans une période où les institutions financières internationales mettent plutôt l'accent sur le remboursement de la dette extérieure et la baisse des effectifs dans la fonction publique, avec un nombre de "diplômés sans emploi", selon l'expression consacrée, qui risque d'augmenter face aux maigres perspectives d'embauche. Or n'est-ce pas les jeunes qui ont plutôt tendance à s'enrôler dans les milices? Par ailleurs, on peut se demander si les chefs de guerre, et partant "leurs petits", toujours armés, qui ont "fait" le roi, ne sentent pas aujourd'hui qu'ils pèsent de moins en moins dans les décisions car menacés par l'arrivée de techniciens dont tous les secteurs de l'administration ont besoin pour remettre le pays en ordre de marche.

Aujourd'hui, très schématiquement, Sassou Nguesso et son entourage se demande à quoi utiliser les excédents pétroliers? A satisfaire les institutions financières internationales pour une reprise de l'aide au développement? A répondre à l'énorme demande intérieure des citoyens congolais ? Ou à continuer à alimenter les "faiseurs de roi", toujours armés, et qui voient désormais leur influence se réduire, leur survie hypothéquée par la "normalisation", et leur vie menacée par l'opération Espoir ? Alors, la mort de Faignond, l'enfant de ce quartier traditionnellement indiscipliné de Poto-Poto, haut dignitaire du régime, faiseur de roi, chef de bande, qui a été célébrée en grande pompe par le régime, n'est-elle pas emblématique de l'impasse actuelle dans laquelle se trouve le régime Sassou Nguesso?

\section{BIBLIOGRAPHIE}

Gruénais M.-E., 1997, "Congo : la fin d'une pseudo-démocratie", Politique Africaine, 68 : 125-133.

Poutier R., 1997, "Les raisons d'une guerre 'incivile"', Afrique contemporaine, 186, avril-juin 1998 :

7-32.

Vallée O., "Les cycles de la dette", Politique Africaine, 31, octobre 1988 : 15-21.

Les journaux

BBC News (http ://news.bbc.co.uk/world/africa), "Ninja clashes in Congo's Capital", 18 décembre 2003.

Cameroon Tribune, "Congo Brazzaville : entraves aux efforts de paix. L'ex-rébellion et le pouvoir se rejettent la responsabilité des actes de violence", 5 janvier 2004, cité par (http :// allAfrica.com).

La Rue Meurt, $n^{\circ} 460,13$ janvier 2004.

Le Défi Africain, $n^{\circ}$ SI, du 13 janvier 2004. 
L'Autre-Vision, $\mathrm{n}^{\circ}$ 56, 1-15 janvier 2004

L 'Humanitaire, n 46, 13-19 janvier 2004.

L'observateur, n’23S, 15-21 janvier 2004.

L'observateur, n² 239,22-28 janvier 2003.

L'observateur, "Le pouvoir de Brazzaville continue de manger ses enfants", n 239, 22-28 janvier 2003.

Tam-Tam d'Afrique, $\mathrm{n}^{\circ}$ 176, 15 janvier 2004.

\section{NOTES}

1.Voilà ce que l'on peut lire sur la pochette de son disque : "Brazzaville, ville d'histoire, capitale de l'Afrique équatoriale française et de la France libre, est une ville d'intégration culturelle et linguistique. Poto-Poto en est le quartier le plus diversifié : Béninois, Sénégalais, Maliens, Togolais, Tchadiens, Camerounais, Centrafricains, Hutus et Tutsi apportent quotidiennement leurs diversités enrichissantes. Poto-Poto est un vrai melting pot dont le produit, débarassé de toute ambigulté ethnique est 'Les enfants de Poto-Poto' Isicl. A travers ce CD, l'orchestre 'Bana Poto-Poto', sous la direction de l'administrateur Maire de Poto-Poto, Bienvenu Faignond, traduit cette manifestation de la diversité des apports musicaux dans la musique congolaise, à Brazzaville, Kinshasa et ne Afrique centrale. Puissiez-vous aimer et faire aimer cet album, afin que le possible de tout un quartier soit possible". A noter que ce texte figure sur une photo représentant un bûcher d'armes en train de brûler avec pour légende "le feu purifie tout".

2.Pour une analyse géo-ethnico-politique du conflit de 1997 à Brazzaville, on pourra se reporter à R. Pourtier.

3.En effet. Ninjas et Cobras se sont violemment opposés. notamment en 1999. Cette rumeur d'alliance entre Ninja et Cobra en 2003 pourrait être crédible, tant les retournements d'alliance ont toujours été spectaculaires dans la vie politique congolaise qui a vu d'anciens condamnés à mort devenir ministres, les pires ennemis d'hier accusés de trafic d'armes, devenir députés, et des alliées d'hier se faire la guerre quelques mois après.

4."Allocution du chef suprême des armées, le général d'armées Denis Sassou Nguesso au réveillon d'armes 2003, le 31 décembre 2003 au mess des officiers de Brazzaville", le journal prenait soin de préciser qu'il s'agissait là d'une transcription de l'Agence Congolaise d'Information, soit l'organe d'information officiel.

5.57 milliards de F. CFA selon les experts internationaux. 30 milliards de F. CFA selon les autorités congolaises.

6.Par exemple. nombreux sont les cadres de la fonction publique. vivant à l'extérieur du pays, ou les employés d'entreprises d'État aujourd'hui disparues. qui. après les guerres. Sont parvenus à faire "rétablir" leur salaire. Attitude certes que l'on peut juger anormale, mais bien compréhensible eu égard à l'importance des pertes subies par tout un chacun à la suite des conflits. Par ailleurs, il est très certainement politiquement délicat pour le régime en place de supprimer les salaires des cadres fonctionnaires résidant à l'étranger ; ceux-ci peuvent toujours constituer une force d'opposition 
disposant d'une audience internationale, et donc dont il est inutile d'accroître le mécontentement.

\section{AUTEUR}

\section{MARC-ERIC GRUÉNAIS}

Directeur de recherche à l'IRD - Université de Provence - Marseille ; gruenais@up.univ-mrs.fr 